\title{
Class wise image retrieval through scalable color descriptor and edge histogram descriptor
}

\author{
Muhammad Imran 1, *, Rathiah Hashim ${ }^{2}$, Aun Irtaz ${ }^{3}$, Azhar Mahmood ${ }^{1}$, Umair Abdullah 4 \\ ${ }^{1}$ Department of Computer Science, SZABIST-ISB, Islamabad, Pakistan \\ 2FSKTM, University Tun Hussin Onn Malaysia, Johor, Malaysia \\ ${ }^{3}$ Department of Computer Science, University of Engineering and Technology Taxila, Punjab, Pakistan \\ ${ }^{4}$ Barani Institute of Information Technology, Rawalpindi, Pakistan
}

\section{ARTICLE INFO}

\section{Article history:}

Received 18 September 2016

Received in revised form

23 November 2016

Accepted 25 November 2016

\section{Keywords:}

Content based image retrieval (CBIR)

SVM

SCD

Edge histogram descriptor

\begin{abstract}
A B S T R A C T
Various domains such as medical science, forensics science and education etc. are generating lot of images on daily bases. As a result of these content generation large image databases are available. These databases are considered as very helpful such as suspects can be searched from forensics database, similarly medical image database can be utilized for the diagnosis purposes. However, proposer management of these databases like, storing and retrieving of images is the demand of the day. Relevant content searching from these databases is a difficult task, however content based image retrieval (CBIR) playing a very important role for searching the relevant contents from these large databases. But this approach is facing some issues. One of the famous issues of CBIR is to describe the image in terms of as feature. This research work aimed is to present a new scheme of image representation by combing the texture and color signature to increase the accuracy of CBIR. Color signatures are generated through Scalable Color Descriptor (SCD) while texture feature are extracted by Edge Histogram Descriptor (EHD). The proposed technique is assessed by testing on the coral image data set and validated by comparing the results with other CBIR approaches.
\end{abstract}

(C) 2016 The Authors. Published by IASE. This is an open access article under the CC BY-NC-ND license (http://creativecommons.org/licenses/by-nc-nd/4.0/).

\section{Introduction}

Due to increasing amount of digital image collections, image storing, searching and browsing are being investigated in present era. Image searching through visual contents is commonly known as Content Based Image Retrieval (CBIR). Various domains such as medical science, forensic science, fabric and fashion design, trade mark design and geographical information management system are using the application of CBIR. CBIR search the images from the image database which are perceptually relevant to the query image. To search the relevant images from the image data bases, CBIR used the visual contents of the images. Shape, color and texture features are used to represent these visual contents. Color is the most frequently used visual pattern in the field of CBIR (Rao et al., 2011a).

\footnotetext{
* Corresponding Author.

Email Address: dr.imran@szabist-isb.edu.pk (M. Imran) https://doi.org/10.21833/ijaas.2016.12.005

2313-626X/@ 2016 The Authors. Published by IASE.

This is an open access article under the CC BY-NC-ND license

(http://creativecommons.org/licenses/by-nc-nd/4.0/)
}

Color information can be represented through color histogram, dominant color descriptor and correlogram. Among these colors histogram is widely used color features. Texture features also play a vital rule to capture the image semantics (Chandankhede et al., 2011).

Representation of image in term of feature vector is the basic step in CBIR which is known as feature extraction which can affect the performance of any CBIR system. Various researchers had proposed different types of types of color and texture features. If the feature vector represents the multiple aspect of the image, then the performance of the CBIR system will be enhanced.

Researchers had proposed the different combination of visual features such as color and texture, color and shape, shape and texture and color, texture and shape in a single feature vector. However, there is still need to explore the image features to have a significant image signature, which can help CBIR to improve its performance.

In this research work author combined the Scalable Color Descriptor (SCD) and Edge Histogram Descriptor (EHD) from MPEG-7, where the aim is to improve the accuracy of CBIR system. The objective 
is to represent the color and texture information of the image into a single feature vector. The SCD is selected due to its interoperability property while EHD is selected to compute the spatial distribution of edges in an image.

Related work is illustrated in section II, a brief description of proposed approach is available in section III, section IV and V are reserved for the experimental detail and results discussion. Finally paper is concluded in section VI.

\section{Related worked}

Fourier Transform was adopted by (Yu et al., 2002) to extract texture information, where color information is represented through first and second moments. Singha et al. (2012) designed a signature by combining color and texture information to enhance the accuracy of CBIR, to extract color features they used color histogram while fast wavelet transform was utilized to extract texture.

This proposed scheme was evaluated through coral data set. Imran et al (2013), adopted color histogram to extract color features. This scheme divides the image into $4 \times 4$ various sub images and then converts every single sub image to HSV color space. Finally computes skewness, variance and mean for $\mathrm{S}, \mathrm{V}$ and $\mathrm{H}$ element of the sub image.

Texture features extracted based on histogram was combined with MPEG-7 color features by Imran et al. (2014).

\section{Proposed approach}

As illustrated above, this paper proposed a scheme to strengthen CBIR. In this scheme, the color and texture feature vector are extracted and combined in sequential order. When user wants to search an image from the image database and used image as a query, system search the relevant images from the image database and ranked according to the top retrievals. If the top retrieval is 10 then first 10 images are selected, if the top retrieval is 20 then 20 top ranked images are selected. From these top ranked images two sets of images are constructed one is relevant and the other is irrelevant. As we are using a bench mark image database, therefore we know the number of images in each class. These two sets of images are further used for the training of Support Vector Machine (SVM). Once SVM is trained, it classifies the similar and non-similar images from the image database and display results to user (Fig. 1).

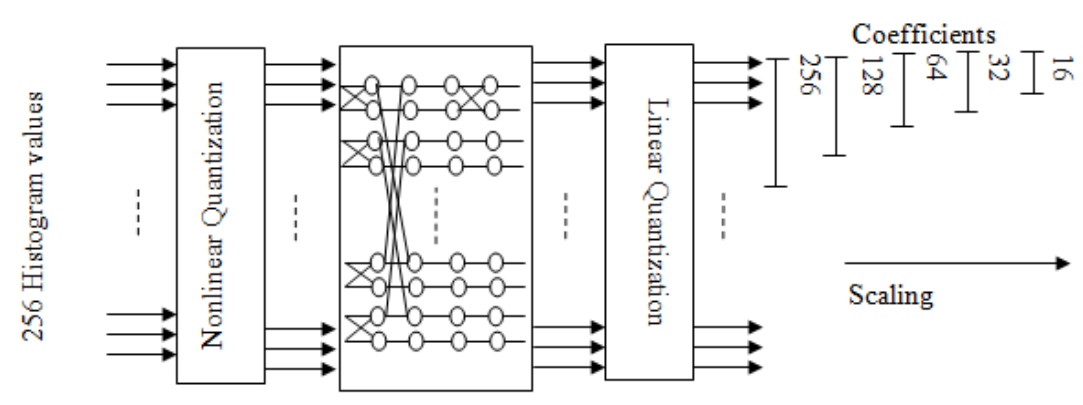

Fig. 1: A schematic diagram of SCD generation

\subsection{Color descriptor}

Scalable Color Descriptor (CSD) address the issue of inter- operability as it fix the the color space to HSV only, with a uniform quantization of the HSV space to 256 bins. The HSV space is quantized homogeneously into a 256 bins, where $\mathrm{H}$ component contains 16 levels, S and V component contains four four levels. the extraction procedure of SCD is illustrated in Fig. 1. The generated output is scalable in terms of num- bers of bins, by altering the number of coefficients used. This scaling property retained the Interoperability between different resolution levels. For detailed SCD extraction method readers are referred to Manjunath et al. (2001).

\subsection{Edge histogram descriptor}

Local edge distribution of the image is represented by the Edge Histogram Descriptor which is the part of MPEG-7 library? The image is divided into $4 \times 4$ sub images as depicted in Fig. 2 and local edge distribution of each sub image is represented through histogram. For the generation of histogram, edges of each sub images are classified into five classes such as horizontal, vertical, 45 diagonal, 135 diagonal and non-directional edges. As the total number of sub images is 16 , therefore $16 \times 5=80$ histogram bins are required. A nondirectional edge and four directional edges are required to detect for the extraction of EHD. All the edges without particular directions are considered as non-directional edges.

These five types of edges are extracted through a block-based edge extraction technique where each sub image is again divided into blocks. The total number of blocks in a sub images is set as constant, despite of the image size, by scaling their size appropriately. One the image is divided into block, each block is categorized into one of the five edge types illustrated above. To classify these block, each block is treated as $2 \times 2$ pixel image and corresponding edge strength is calculated by apply the appropriate edge detector. The image blocks exceeding the edge strength a certain minimum threshold are used to compute the histogram. 
Thus for each image block, five edge strength are computed, one for each of the five filters as shown in Fig. 3. A block is treated as edge block, if the strength of the corresponding block exceeds a preset threshold value. Once a block is treated as edge it contributes to the histogram bins.

\section{Experimental detail}

Experiments are performed on coral image database for assessing and validating the proposed Scheme. Coral database is a benchmark image database and it has two version. One version containing 9908 images while other version has 1000 images. Each version has images from from 10 different categorizes and each category contains 100 images.

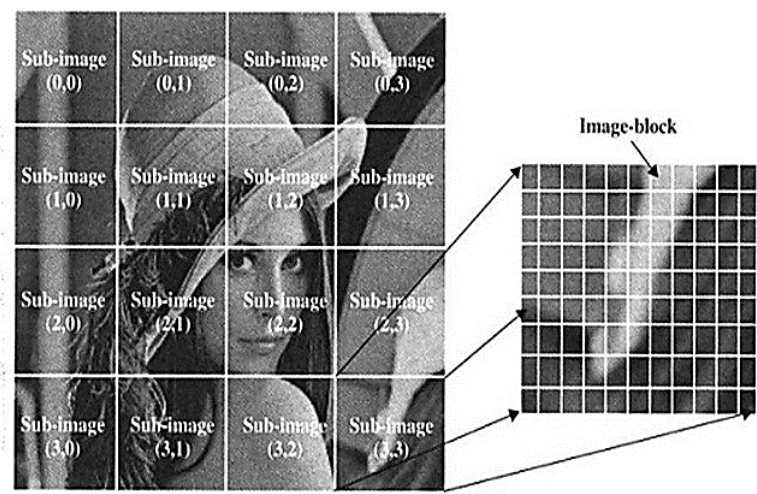

Fig. 2: Definition of sub image and image-blocks

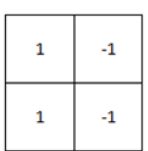

(a)

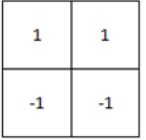

(b)

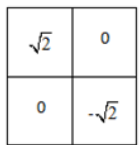

(c)

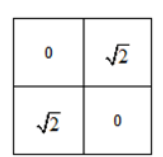

(d)

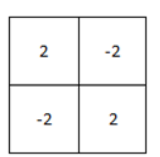

(e)
Fig. 3: A schematic diagram of SCD generation

The classes are; African people, Elephants, , Buses, Beach, Flowers, Dinosaurs, Buildings, Mountains, Horses and Food. Based on literature, recall, precision and their harmonic means was selected to evaluate the proposed scheme. The of precision, recall and $\mathrm{F}$-score is from $0-1$, where 0 means $0 \%$ accuracy rate and 1 means $100 \%$ accuracy rate. The Following equations are used to compute these performance metrics.

$$
\begin{aligned}
& \text { Precision }=\frac{\text { No.of True Positive }}{\text { No.of True Poisitive }+ \text { False Positive }} \\
& \text { Recall }=\frac{\text { No. of True Positive }}{\text { Total No.of True Positive }} \\
& F-\text { Score }=2 \times\left(\frac{\text { Precision.Recall }}{\text { Precision }+ \text { Recall }}\right)
\end{aligned}
$$

To assess the proposed signature scheme, 15 images were selected from each category randomly as query image. Various types of experiments were conducted such as to retrieval 10 most relevant images, 20 most relevant images, 30 and 50 most relevant images, which are known as top 10, 20, 30 and 50 respectively; for each experiment, average recall, F-Score and average precision were recorded.

\section{Results}

The performance of proposed scheme in term of, average Recall, average precision and F-Score is shown in the Table 1 to Table 3 respectively.

Table 1: Average precision of roposed approach for $n$ top retrievals

\begin{tabular}{|c|c|c|c|c|} 
Class & $\mathrm{n}=10$ & $\mathrm{n}=20$ & $\mathrm{n}=30$ & $\mathrm{n}=50$ \\
Africa & 0.9 & 0.98 & 0.92 & 0.89 \\
\cline { 2 - 5 } Beach & 0.57 & 0.58 & 0.52 & 0.57 \\
Buildings & 0.74 & 0.82 & 0.83 & 0.59 \\
Buses & 0.89 & 0.95 & 0.95 & 0.94 \\
\hline Dinosaurs & 1 & 1 & 0.96 & 1 \\
Elephant & 0.86 & 0.65 & 0.47 & 0.38 \\
Flower & 1 & 0.95 & .89 & 0.78 \\
Horses & .93 & 1 & .99 & .91 \\
Mountain & .58 & .43 & .41 & .35 \\
Food & .92 & .88 & .84 & .8 \\
\hline Avg & .839 & .824 & .778 & .72 \\
\hline
\end{tabular}

Table 2: Average recall ofoposed approach for $\mathrm{n}$ top retrievals

\begin{tabular}{|c|c|c|c|c|} 
Class & $\mathrm{n}=10$ & $\mathrm{n}=20$ & $\mathrm{n}=30$ & $\mathrm{n}=50$ \\
Africa & .16 & .32 & .42 & .59 \\
\cline { 2 - 5 } Beach & .1 & .19 & .24 & .38 \\
Buildings & .13 & .27 & .38 & .39 \\
Buses & .16 & .31 & .44 & .62 \\
Dinosaurs & .18 & .33 & .44 & .66 \\
Elephant & .15 & .21 & .22 & .25 \\
\cline { 2 - 5 } Flower & .18 & .31 & .41 & .52 \\
Horses & .16 & .33 & .46 & .6 \\
Mountain & .1 & .14 & .19 & .23 \\
\hline Food & .16 & .29 & .39 & .53 \\
Avg & .148 & .27 & .359 & .477 \\
\hline
\end{tabular}

Table 3: Average f-score of roposed approach for $\mathrm{n}$ top retrievals

\begin{tabular}{|cc|c|c|c|}
\hline Class & $\mathrm{n}=10$ & $\mathrm{n}=20$ & $\mathrm{n}=30$ & $\mathrm{n}=50$ \\
Africa & 0.09 & 0.19 & 0.27 & 0.44 \\
\cline { 2 - 5 } Beach & 0.05 & 0.11 & 0.15 & 0.28 \\
\hline Buildings & .07 & .16 & .25 & .29 \\
Buses & .08 & .19 & .28 & .47 \\
Dinosaurs & .1 & .2 & .29 & .5 \\
\hline $\begin{array}{c}\text { Elephant } \\
\text { Flower }\end{array}$ & .08 & .13 & .14 & .19 \\
Horses & .09 & .19 & .26 & .39 \\
\hline Mountain & .05 & .08 & .29 & .45 \\
Food & .09 & .17. & .25 & .17 \\
Avg & .08 & .16 & .23 & .35 \\
\hline
\end{tabular}

Category wise performance comparisons of proposed approach with previous approaches in term of average precision are shown in Table 4 and Fig. 4 depicts the same result in graphical form. For further validation, we also calculate the F-Score and results of F-Score are compared with previous techniques in Table 5 and graphically presented in Fig. 5.

\section{Analysis}

Table 4 and Fig. 4, proved that precision value achieved by the proposed feature extraction method 
is higher as compared to Rao et al. (2011b), Huang and Dai (2003), ICTEDCT (Youssef, 2012), IGA (Lai and Chen, 2011), and CTDCIRS (Rao et al., 2011a). Overall average precision achieved by proposed approach is 0.82 which means that 82 was achieved while Huang, CTDCIRS, Babu Rao, ICTEDCT and IGA achieved $53 \%, 70 \%, 60 \%, 81 \%$ and $80 \%$ accuracy rate.

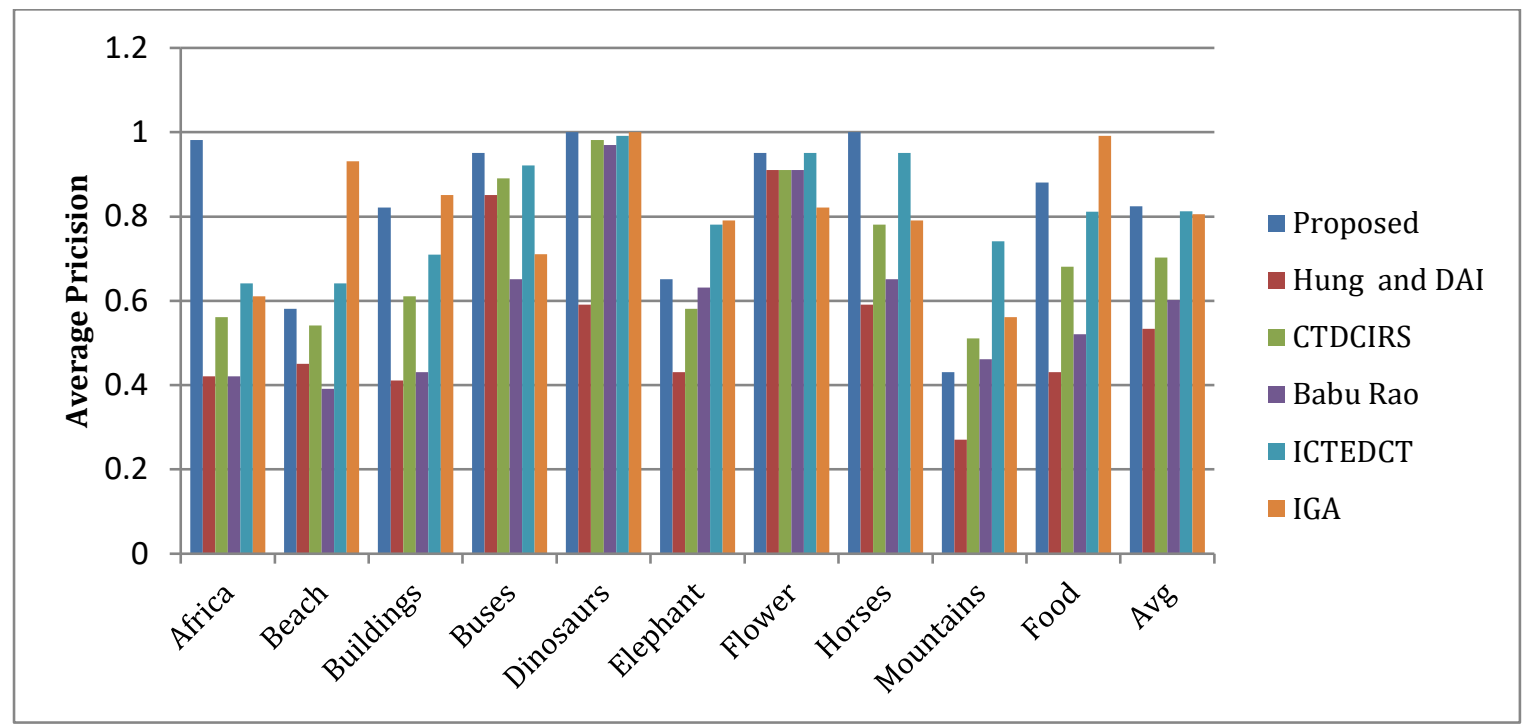

Fig. 4: Class wise performance comparison based on precision

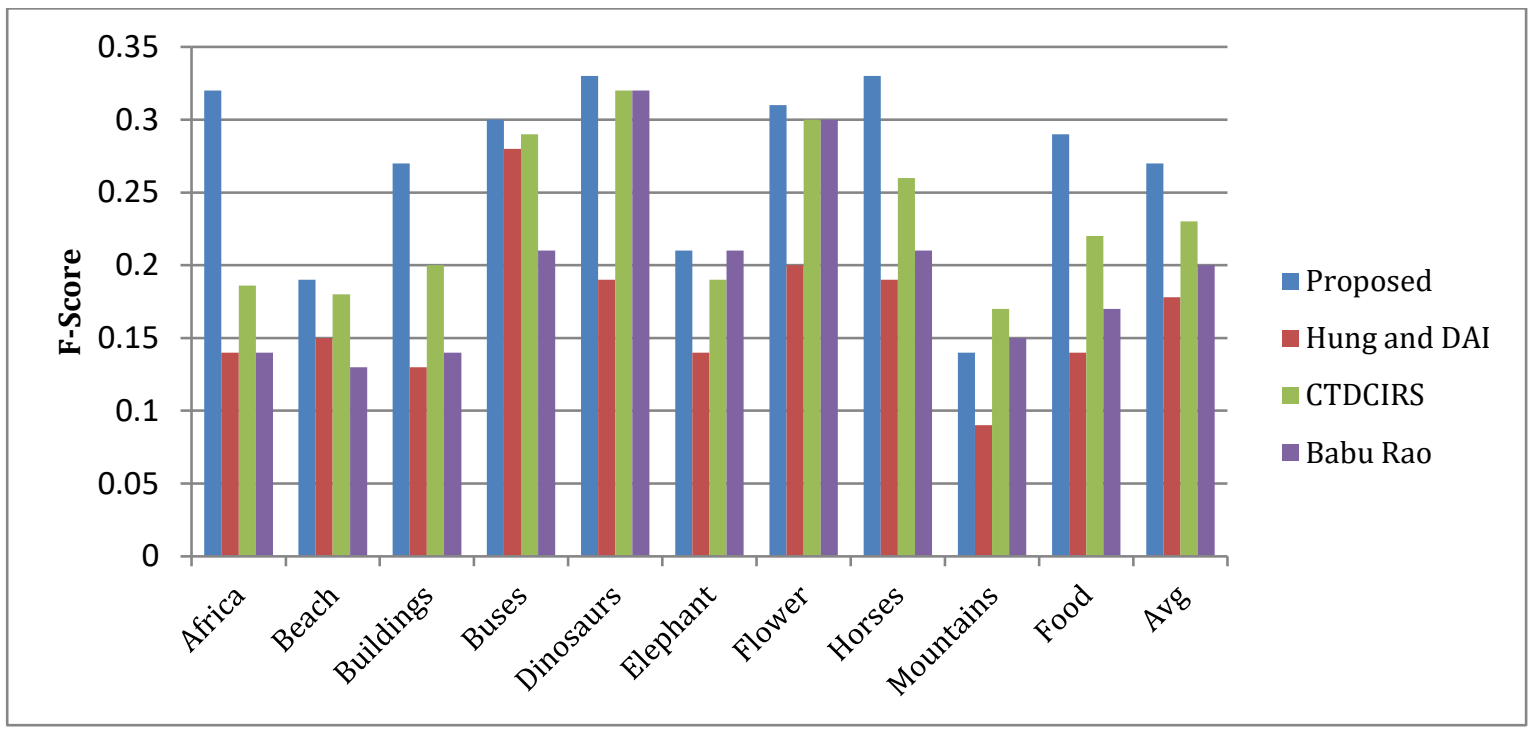

Fig. 5: Class wise performance comparison based on F- Score

Table 4: Erformance comparison of proposed approach with previous approaches in term of precision

\begin{tabular}{|cc|c|c|c|c|c|}
\hline Class & Proposed & Hung and DAI & \multicolumn{1}{c}{ CTDCIRS } & Babu Rao & ICTEDCT & IGA \\
Africa & 0.981 & 0.421 & 0.561 & 0.421 & 0.641 & 0.611 \\
Beach & 0.581 & 0.451 & 0.541 & 0.391 & 0.641 & 0.931 \\
Buildings & 0.821 & 0.411 & 0.611 & 0.431 & 0.71 & 0.851 \\
Buses & 0.951 & 0.851 & 0.891 & 0.651 & 0.921 & 0.711 \\
Dinosaurs & 1 & 0.591 & 0.981 & 0.97 & 0.991 & 1 \\
Elephant & 0.651 & 0.431 & 0.581 & 0.631 & 0.781 & 0.791 \\
Flower & 0.951 & 0.91 & 0.91 & 0.91 & 0.951 & 0.821 \\
Horses & 1 & 0.591 & 0.781 & 0.651 & 0.951 & 0.791 \\
Mountains & 0.431 & 0.271 & 0.511 & 0.461 & 0.741 & 0.561 \\
Food & 0.881 & 0.431 & 0.681 & 0.521 & 0.811 & 0.991 \\
Avg & 0.824 & 0.534 & 0.703 & 0.602 & 0.812 & 0.805 \\
\hline
\end{tabular}

Only ICTEDCT and IGA among all competitors perform well but still their accuracy rate is below proposed approach. Performance comparison based on F-Score is illustrated in Table 5 and Fig. 5, where it is very clear that the F-Score achieved by proposed approach is higher than the previous approaches.

The average precision achieved by proposed approach for all the classes is higher than $60 \%$ except mountain class where the average precision is 
43\% while Hung and Dai method achieved higher than $60 \%$ accuracy rate only for one class which is bus class. From the given result it is clear that the proposed approach is more stable and robust as compared to other competitors.

\section{Conclusion}

To overcome the shortcomings of CBIR, this paper presented a novel signature scheme to strengthen the performance of CBIR. In this proposed approach color and texture features are combined. Scalable Color Descriptor and Edge Histogram Descriptors are adopted to extract color and texture features respectively. It is observed from the obtained results that, presented scheme achieved better performance than the various existing CBIR's approaches.

Table 5: Performance comparison of proposed approach with previous approaches in term of f-score

\begin{tabular}{ccc|c|c|c|}
\hline Class & Proposed & Hung and DAI & CTDCIRS & Babu Rao \\
Africa & 0.32 & .14 & .186 & .14 \\
Beach & .19 & .15 & .18 & .13 \\
\cline { 2 - 5 } Buildings & .27 & .13 & .20 & .14 \\
Buses & .3 & .28 & .29 & .21 \\
Dinosaurs & .33 & .19 & .32 & .32 \\
Elephant & .21 & .14 & .19 & .21 \\
\cline { 2 - 5 } Flower & .31 & .2 & .3 & .3 \\
Horses & .33 & .19 & .26 & .21 \\
Mountains & .14 & .09 & .17 & .15 \\
Food & .29 & .14 & .22 & .17 \\
Avg & .27 & .178 & .23 & .2 \\
\hline
\end{tabular}

\section{Acknowledgements}

The authors would like to thank Universiti Tun Hussein Onn Malaysia for supporting this research work under the MDR Grant Vote 1315 and Shaheed Zulfiqar Ali Bhutto Institute of Science and Information Technology for providing the platform to conduct this research work.

\section{References}

Chandankhede PH, Puranik PV and Bajaj PR (2011). Soft computing tool approach for texture classification using Discrete Cosine Transform. In the $3^{\text {rd }}$ IEEE International Conference, Electronics Computer Technology (ICECT): 296-299. https://doi.org/10.1109/ICECTECH.2011.59419 07

Huang PW and Dai SK (2003). Image retrieval by texture similarity. Pattern Recognition, 36(3): 665-679.

Imran M, Hashim R and Khalid NEA (2013). New approach to image retrieval based on color histogram. In the $4^{\text {th }}$ International Conference on Advances in Swarm Intelligence, ICSI, Harbin, China: 453-462. https://doi.org/10.1007/978-3642-38715-9-54

Imran M, Hashim R and Khalid NEA (2014). Content based image retrieval using MPEG-7 and histogram. In the $1^{\text {st }}$ International Conference on Soft Computing and Data Mining, SCDM, Parit Raja, Batu Pahat, Malaysia: 453-466. https://doi.org/10.1007/978-3-319-07692-8_43
Lai CC and Chen YC (2011). A user-oriented image retrieval system based on interactive genetic algorithm. IEEE Transactions on Instrumentation and Measurement, 60(10): 3318-3325.

Manjunath BS, Ohm JR, Vasudevan VV and Yamada A (2001). Color and texture descriptors. IEEE Transactions on Circuits and Systems for Video Technology, 11(6): 703-715.

Rao MB, Rao BP and Govardhan A (2011a). CTDCIRS: content based image retrieval system based on dominant color and texture features. International Journal of Computer Applications, 18(6): 40-46.

Rao MB, Rao DBP and Govardhan A (2011b). Content based image retrieval using dominant color, texture and shape. International Journal of Engineering Science and Technology (IJEST), 3(4): 2887-2896.

Singha M, Hemachandran K and Paul A (2012). Content-based image retrieval using the combination of the fast wavelet transformation and the colour histogram. IET Image Processing, 6(9): 1221-1226.

Youssef SM (2012). ICTEDCT-CBIR: Integrating curvelet transform with enhanced dominant colors extraction and texture analysis for efficient content-based image retrieval. Computers and Electrical Engineering, 38(5): 1358-1376.

Yu H, Li M, Zhang HJ and Feng J (2002). Color texture moments for content-based image retrieval. In the IEEE Proceedings of International Conference on Image Processing: 929-932. https://doi.org/10.1109/ICIP.2002.1039125 